\title{
The Examination, by Partition Paper Chromatography, of the Nitrogen Metabolism of Bacteria
}

\author{
BY H. PROOM AND A. J. WOIWOD \\ The Wellcome Reseurch Laboratories, Beckenham, Kent
}

\begin{abstract}
SLMMARY: Some 300 strains of bacteria representing twenty genera were grown on an acid-hydrolysed casein medium. Using the paper chromatographic technique of Woiwod (1949) a preliminary survey was made of the amino-acid and polypeptide composition of the bacterial culture filtrates. It was proved that: $(a)$ changes may occur in the filtrate chromatogram which have group or species significance; $(b)$ bacteria with simple nutrient requirements, i.e. which utilize ammonia, do not affect the chromatogram in the initial growth stage; $(c)$ whenever the chromatogram is affected serine is the first amino-acid to be metabolized; $(d)$ Gram-positive bacteria eliminate the aspartic acid spot but leave the basic amino-acid group unaffected, and the reverse occurs with Gram-negative bacteria ; $(e)$ many bacteria synthesize ninhydrinpositive material, presumably polypeptide, the synthesis of a given kind of polypeptide being sometimes associated with a particular group or species of bacteria. By a disintegrator technique it was demonstrated that bacteria store many free aminoacids inside the cell.
\end{abstract}

The application of partition paper chromatography to the qualitative analysis of complex mixtures of amino-acids and polypeptides by Consden, Gordon \& Martin (1944) has a general appeal because it is an attractive and simple method which has no parallel in the classical methods of protein analysis. Within the limitations of the method it is possible, in a reasonable time, to obtain a general picture of some of the aspects of the nitrogen metabolism of bacteria grown in a selected medium; and also to examine a sufficient number of cultures to distinguish strain, species and group differences. We have made a general survey of the amino-acid and polypeptide metabolism of some 300 strains of bacteria, representing twenty genera, grown on a casein hydrolysate medium. The purpose of this paper is to describe the general picture and the problems which have arisen out of this survey. The more detailed examination of selected problems will be the subject of further studies.

\section{METHODS}

Medium. Bacteria were grown in a liquid medium consisting of an acidhydrolysate of casein ( $3 \mathrm{~g} . \mathrm{N} / \mathrm{l}$.), yeast extract, growth factors and metallic salts with $0 \cdot 1 \%$ added glucose. The medium was arbitrarily selected with the object of giving clear chromatograms uncomplicated by the initial presence of polypeptide or protein. The amount of glucose was kept to a minimum in order to prevent $\mathrm{pH}$ changes due to acid production during growth. The growth factors, yeast extract and metallic salts were added in an attempt to extend the range of bacteria which would grow in the medium. The exact composition and preparation of the medium is as follows. 
Casein acid-hydrolysate $\equiv \mathbf{3}$ g. $\mathbf{N} / \mathbf{l}$.

Yeast extract, $100 \mathrm{ml}$.

Glucose, $1.0 \mathrm{~g}$.

$\mathrm{NaCl} \equiv 0.5 \%$

Tryptophan $\mathbf{0} \cdot \mathbf{2} \mathrm{g}$.

$\mathrm{MgSO}_{4} 7 \mathrm{H}_{2} \mathrm{O}, 0.45 \mathrm{~g}$.

$\mathrm{CuSO}_{4} .5 \mathrm{H}_{2} \mathrm{O}, 0.01 \mathrm{~g}$.

$\mathrm{ZnSO}_{4} \cdot 7 \mathrm{H}_{2} \mathrm{O}, 0.008 \mathrm{~g}$.

$\mathrm{MnCl}_{2} .4 \mathrm{H}_{2} \mathrm{O}, 0.003 \mathrm{~g}$.

Riboflavin, $200 \mu \mathrm{g}$.
Calcium pantothenate, $400 \mu \mathrm{g}$.

Biotin, $4 \mu \mathrm{g}$.

Aneurin, $200 \mu \mathrm{g}$.

Uracil, $15 \mathrm{mg}$.

Adenine, $15 \mathrm{mg}$.

Pyridoxin $\mathrm{HCl}, 1 \cdot 2 \mathrm{mg}$.

$p$-Aminobenzoic acid, $40 \mu \mathrm{g}$.

Nicotinic acid, $600 \mu \mathrm{g}$.

Choline chloride, $7 \cdot 5 \mathrm{mg}$.

Pteroylglutamic acid, $\mathbf{0 \cdot 2} \mu \mathrm{g}$.

\section{Distilled water to 11 .}

The casein hydrolysate was prepared by hydrolysing Glaxo light white soluble casein with $c .6 \mathrm{~N}$ hydrochloric acid. Total nitrogen determinations were made on the concentrated digest in order that the nitrogen content of the final medium could be adjusted to $3 \mathrm{~g}$./ 1 . The yeast extract was prepared by boiling $500 \mathrm{~g}$. bakers' yeast with 2 l. of distilled water for $30 \mathrm{~min}$. and filtering through paper. The growth factors and metallic salts were added as convenient volumes of stock solutions. The medium was made up to the required volume with distilled water and the $\mathrm{pH}$ adjusted to $7 \cdot 6$. The medium was heated to $95^{\circ}$, sterilized by filtration through a Berkefeld-type candle and filled aseptically into suitable containers which were then incubated to test for sterility.

Growth. The medium was dispensed in $20 \mathrm{mI}$. lots in $1 \mathrm{oz}$. screw-capped bottles, and where necessary anaerobiosis was established by the addition of $0.01 \%$ thiolacetic acid. The medium was seeded with a loopful of culture from the appropriate solid medium or with a few drops of a suitable liquid culture. The cultures were incubated at $37^{\circ}$ for 3 days and then were divided into two parts. A $5 \mathrm{ml}$. portion was removed into a sterile screw-capped bottle and incubated for a further 7 days. To the remainder were added a few drops of bromocresol purple and sterile $\mathrm{N}-\mathrm{HCl}$ until a yellow colour developed. Acetate buffer ( $3 \mathrm{ml}$. of $\mathrm{m} / 25)$ was added to give a final $\mathrm{pH}$ of 4.5 and the culture incubated for a further 7 days at $37^{\circ}$. These periods were selected arbitrarily, on the basis of preliminary experiments, to distinguish changes that take place in the medium during the active growth stage and changes developing after growth has almost ceased. In this medium during the growth of any of the organisms examined the reaction was either unaltered or alkaline $(\mathrm{pH} 7 \cdot 6-9 \cdot 0)$, so that the effects observed were those taking place at slightly alkaline reaction. In order to examine effects due to enzyme activity at acid reaction, part of the culture was adjusted to the arbitrarily selected $\mathrm{pH}$ of $4 \cdot 5$, as described above. Unless otherwise stated in the text, cultures were always grown in this way.

Chromatographic analysis. Culture filtrates were examined by Woiwod's (1949) technique. The solvent used for one-dimensional paper chromatography was $n$-butanol + acetic acid mixture; phenol was used as the second solvent for two-dimensional work. In each case the stationary phase was water. The chromatograms were run on sheets of Whatman No. 4 paper $\left(22 \frac{1}{2} \times 18 \frac{1}{4}\right.$ in.). Each filtrate was tested in duplicate and ten filtrates were examined on each 
sheet of paper. At each end of the series of spots on the paper the uninoculated medium was always included as a control. The chromatograms, after development, were examined, the results noted and a permanent photographic record taken. Pl. 1, fig. 1, shows a one-dimensional chromatogram of the control medium, with a key showing the position of the various amino-acids present in the casein digest.

\section{RESULTS}

\section{Removal of amino-acids during the first three days of growth}

The chromatographic picture of the filtrates from a culture after 3 days' growth may differ in a number of ways from that of the control medium. The effects are illustrated in Pl. 1, fig. 2. With some organisms, such as Pseudomonas aeruginosa and Bacillus subtilis, the chromatogram is unaffected in spite of heavy growth. With our technique no definite conclusions can be drawn about the removal of amino-acids from the medium for the direct building of bacterial proteins, since in most of the growths obtained the nitrogen of the cells did not represent more than $3 \%$ of the total nitrogen initially present in the medium. With $P$ s. aeruginosa, however, during the first 3 days an opacity equivalent to $15 \times 10^{9}$ organisms $/ \mathrm{ml}$, or $0 \cdot 3 \mathrm{~g}$. $\mathrm{N} / \mathrm{l}$., was obtained by shaking flask cultures. This amount of nitrogen corresponded to about $10 \%$ of that initially present in the medium, and had it been taken exclusively from the amino-acid constituents it would almost certainly have been visible on the chromatogram.

With bacteria requiring more complex nutrients, i.e. specific amino-acids and other essential metabolites, a change in the chromatogram always takes place during the early growth. This is illustrated in Pl. 1, fig. 2 with Streptococcus pyogenes, Staphylococcus aureus, Corynebacterium diphtheriae, Shigella dysenteriae (Shiga), Escherichia coli, Pasteurella boviseptica, Serratia marcescens and Proteus vulgaris. In all cases the serine spot decreases in intensity, and in many the glycine spot is unaffected. This is also illustrated in Pl. 1, fig. 3, which shows the decrease in serine with ten strains of Shigella paradysenteriae (Boyd types; Sh. boyd). This plate also shows that the effect is clearly defined and occurs to an equal extent with a number of different strains. During this survey we confined our attention to changes of this order, which are not only unequivocal but are not subject to strain variation. With many Gram-negative organisms the aspartic acid spot increases in intensity; in Pl. 1,fi g. 2, the increase with Esch. coli and Ser. marcescens is very evident. This increase proved, in some cases at least, to be due to the formation of polypeptides.

A number of Gram-negative species metabolize the basic amino-acids. This is illustrated in Pl. 1, fig. 2, by Ser. marcescens. Vibrio comma does so markedly, the basic amino-acid group often being completely eliminated within 2 days. With $\operatorname{Pr}$. vulgaris the valine and leucine groups are attacked; with other organisms these groups are not usually metabolized during the first 3 days. With many Gram-positive organisms the aspartic acid spot disappears from the chromatogram (Pl. 1, fig. 2, with Str. pyogenes, Staph. aureus and Coryne. diphtheriae). A list of the groups of organisms tested and the aminoacids removed during the first 3 days' growth is given in Table 1. In most cases 
at least ten strains were examined in each group. Differences between species within a group were also observed, and the use of more suitable media is expected to reveal many others. For example, in medium enriched with soluble starch Haemophilus parapertussis, but not $\boldsymbol{H}$. pertussis, attacked the leucine and valine groups.

Table 1. The effect of growth of organisms of different genera on the main aminoacid spots as they appear on the single-dimensional chromatogram

\begin{tabular}{|c|c|c|c|c|c|c|c|c|c|c|c|c|c|c|c|c|c|c|c|c|c|}
\hline \multicolumn{2}{|c|}{$\begin{array}{l}\text { Period of } \\
\text { incubation ... }\end{array}$} & \multicolumn{6}{|c|}{$\begin{array}{l}3 \text { days at } \\
\text { alkaline reaction }\end{array}$} & \multicolumn{7}{|c|}{$\begin{array}{l}10 \text { days at } \\
\text { alkaline reaction }\end{array}$} & \multicolumn{7}{|c|}{$\begin{array}{l}3 \text { days at alkaline and } \\
7 \text { days at acid reaction }\end{array}$} \\
\hline Genus & 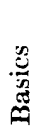 & $\begin{array}{l}\frac{0}{\hat{E}} \\
\frac{\tilde{E}}{2} \\
\frac{0}{4}\end{array}$ & 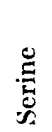 & .气 & $\stackrel{\stackrel{\Xi}{\Xi}}{\stackrel{\Xi}{Z}}$ & 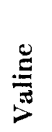 & & 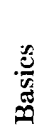 & 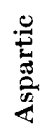 & 芯 & 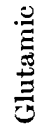 & $\underset{\Xi}{\stackrel{\Xi}{\Xi}}$ & $\stackrel{\mathscr{\Xi}}{\stackrel{\Xi}{\pi}}$ & 导 & 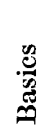 & 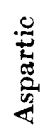 & 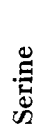 & 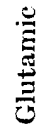 & 苛 & 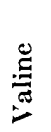 & \\
\hline Pseudomonas & - & - & - & - & - & - & - & + & + & + & + & + & + & + & + & + & + & + & + & + & + \\
\hline Vibrio & + & - & + & - & - & - & - & + & - & + & + & $x$ & - & - & + & - & + & + & + & - & - \\
\hline Neisseria & - & - & + & - & - & - & - & $x$ & - & + & + & - & - & - & + & - & + & + & - & - & - \\
\hline Alkaligenes & - & - & - & - & - & - & - & $x$ & - & + & + & - & - & - & + & - & + & + & - & - & - \\
\hline Escherichia & $x$ & - & + & - & - & - & - & + & - & + & + & $\times$ & - & - & + & + & + & + & + & + & + \\
\hline Aerobacter & $x$ & - & + & - & - & - & - & + & - & + & + & - & - & - & + & + & + & + & + & + & + \\
\hline Klebsiella & + & - & + & - & - & - & - & + & - & + & + & - & - & - & + & - & + & + & + & + & + \\
\hline Serratia & + & - & + & - & - & - & - & + & - & + & + & $x$ & - & - & + & - & + & + & + & + & + \\
\hline Proteus & - & - & + & - & - & + & + & + & + & + & + & + & + & + & + & + & + & + & + & + & + \\
\hline Salmonella & $x$ & - & + & - & - & - & - & + & - & + & + & - & - & - & + & - & + & + & $x$ & + & + \\
\hline Shigella & - & - & + & - & - & - & - & + & - & + & + & - & - & - & + & - & + & + & + & - & - \\
\hline Pasteurella & - & - & + & - & - & - & - & + & - & + & + & - & - & - & - & - & + & + & - & - & - \\
\hline Haemophilus & - & - & + & - & - & $x$ & $x$ & + & - & + & + & - & + & + & + & - & + & + & + & $x$ & $x$ \\
\hline Bacillus & - & - & - & - & - & - & - & - & + & + & + & + & + & + & $x$ & + & + & + & + & + & + \\
\hline Mycobacterium & - & - & - & - & - & - & - & - & + & + & + & - & - & - & - & + & + & + & - & - & - \\
\hline Staphylococcus & - & + & + & - & $x$ & - & - & - & + & + & + & + & - & - & - & + & + & + & + & - & - \\
\hline Diplococcus & - & + & + & - & - & - & - & - & + & + & + & + & - & - & - & + & + & + & + & - & - \\
\hline Streptococcus & - & + & + & - & - & - & - & - & + & + & + & $x$ & - & - & - & + & + & + & $x$ & - & - \\
\hline Lactobacillus & - & + & + & - & - & - & - & - & + & + & + & $x$ & - & - & - & + & + & + & $x$ & - & - \\
\hline Corynebacterium & - & $x$ & + & - & - & - & - & - & + & + & + & - & - & - & - & + & + & + & - & - & - \\
\hline Clostridium & - & $x$ & + & + & - & - & - & - & + & + & + & - & - & - & - & + & + & + & - & - & - \\
\hline & & & & & & & & & & & & & & & & & & & & & \\
\hline
\end{tabular}

\section{Removal of amino-acids during prolonged incubation at acid or alkaline reaction}

After prolonged incubation at $37^{\circ}$, at either acid or alkaline reaction, a further series of changes may occur in the chromatogram. The changes are usually more marked at the acid reaction and may be so extensive that all ninhydrinpositive material is removed from the chromatogram. From the summary of the results in Table 1, it is clear that changes occurring during the first 3 days usually progress to near or complete extinction of the corresponding aminoacid spot. Organisms, such as $\boldsymbol{B}$. subtilis or $\boldsymbol{P s}$. aeruginosa, that have simple nutrient requirements and which initially cause little change in a chromatogram, may produce most extensive changes on further incubation. There is a sharply 
defined difference between Gram-positive and Gram-negative organisms. The former do not attack the basic amino-acid group but remove aspartic.acid early, whereas the latter produces the reverse effect.

A series of chromatograms of the filtrates of bacterial cultures taken at intervals gives an overall picture of the succession of changes taking place in the medium-a picture that can hardly be obtained by any other method. Pl. 2, fig. 2, shows the effect of incubation at acid reaction on a number of cultures of $\boldsymbol{E s c h}$. coli. The following changes can be seen: elimination of serine and decrease in basic amino-acid group (strain 2); elimination of alanine, glutamic acid and the basic amino-acid group (strains $4,7,1,3$ ); appearance of polypeptide in the aspartic acid position (strains $5,7,9$ ); appearance of polypeptide near the tyrosine position (strains 1, 3-9); decrease in the valine group and the leucine group with polypeptides in the aspartic acid and tyrosine positions (strain 6); finally, almost complete elimination of ninhydrin-positive material from the chromatogram, only traces of the two polypeptides being left (strain 8). If samples from strain 8, for example, had been taken at intervals during incubation the changes would have occurred in the above order. It should be noted that in Pl. 2, fig. 2, there is a drift in the chromatogram, due to irregularities during the solvent-run, which on casual examination might lead to misinterpretation. Thus with strain 4 the glutamic acid spot might appear to be in the alanine position; however, closer examination of the chromatogram reveals the nature and extent of the drift.

\section{Possible synthesis of polypeptides}

During the examination of the filtrates from some species a number of ninhydrin spots appeared on the chromatogram which were not present in the basal medium. Since they did not correspond in position, and in some cases in ninhydrin colour, to any of the amino-acids present in the basal medium, we suggest that they are likely to be polypeptides. In the case of $\mathrm{Cl}$. tetani one such ninhydrin-positive spot which appeared on the chromatogram below the leucine group (Pl. 2, fig. 1 (3)), was eluted, acid-hydrolysed and shown by two-dimensional chromatography to be a polypeptide. In other cases the polypeptide nature of these spots has not yet been unequivocally demonstrated. These presumed polypeptides were often different for different species since they appeared in various positions on the chromatogram. The effect is illustrated in Pl. 2, fig. 1. They may appear during the early growth stage, as with $\boldsymbol{P r}$. vulgaris and $\mathrm{Cl}$. tetani, or later after growth has ceased, as with Esch. coli and $S h$. dysenteriae. They may appear at alkaline reaction or only at acid reaction and their appearance is always associated with the disappearance of a particular amino-acid from the chromatogram. This associated amino-acid may be different for different species; for example, it is glutamic acid with $\mathrm{Cl}$. tetani (Pl. 2, fig. 1 (3)), leucine group with Pr. vulgaris (Pl. 2, fig. 1 (5)), alanine with Staph. aureus and proline with $\mathrm{Cl}$. bifermentans (Pl. 2, fig. 1 (1)). The association between the amino-acids and the production of 'polypeptides' is evident, for when the particular amino-acid was not removed no 'polypeptide' appeared 
and, moreover, the strength of the 'polypeptide' spot varied inversely with the strength of the amino-acid spot. This inverse relationship is clearly shown in Pl. 2, fig. 2, with nine strains of Esch. coli. The 'polypeptide' spot, which appears just above the tyrosine position on the chromatogram, is absent from the control medium, very intense with strains 1,3 and 5 when the glutamic acid has disappeared, and just visible with strains 2 and 4 where the glutamic acid is relatively unaffected.

Table 2. Synthesis of polypeptides by bacteria, showing the position of the polypeptide spot on the chromatogram, the amino-acid which is associated with the production of the polypeptide and the reaction at which the polypeptide is formed

$\quad$ Genus or spect
Aerobacter, Escherid
Salmonella, Ser
Shigella
Escherichia, Shigelt
Proteus
Staphylococcus
Cl. tetani
Cl. bifermentans
Cl. sordellii

\begin{tabular}{|c|c|c|}
\hline $\begin{array}{l}\text { Position of polypeptide } \\
\text { on chromatogram }\end{array}$ & $\begin{array}{c}\text { Associated } \\
\text { amino-acids }\end{array}$ & $\begin{array}{l}\text { Reaction } \\
\text { of filtrate }\end{array}$ \\
\hline On aspartic acid spot & Serine & Alkaline \\
\hline On tyrosine spot & Glutamic & Acid \\
\hline $\begin{array}{l}\text { Two polypeptides after } \\
\text { leucine group }\end{array}$ & $\begin{array}{l}\text { Leucine } \\
\text { group }\end{array}$ & Alkaline \\
\hline Before tyrosine spot & Alanine & Alkaline \\
\hline After leucine group & Glutamic & Alkaline \\
\hline $\begin{array}{l}\text { Two polypeptides one } \\
\text { before tyrosine, one } \\
\text { on valine group }\end{array}$ & Proline & Alkaline \\
\hline
\end{tabular}

The hydrolysis of the $C l$. tetani polypeptide showed that it was not a polymer of the associated glutamic acid nor had it an exceptionally high glutamic acid content. Table 2 lists the 'polypeptides' so far observed which give intense spots on the chromatogram. In addition to these, many indefinite spots suggesting 'polypeptide' formation have been observed; further examination with more favourable media is likely to show many others.

\section{Amino-acids and polypeptides inside the cell}

Using a tissue disintegrator (supplied by H. Mickle, Romeyn Works, Romeyn Road, London, S.W. 16) and shaking a dense suspension of washed organisms with small glass beads (Ballotini Grade 9), as described by Elliot \& Gale (1948), the storage of ninhydrin-positive material inside the cell is being examined. Some of the preliminary results are shown in $\mathrm{Pl}$. 2, fig. 3. It can be seen that many amino-acids are stored within the cells. The basic amino-acid group, aspartic acid, serine, glutamic acid, the valine and leucine groups are clearly visible in the chromatogram of disintegrated Staph. aureus. With the exception of glutamic acid these are also visible in the disintegrate of Esch. coli. There is little evidence of the presence of polypeptides.

\section{Hydrolysis of whole bacteria}

The organisms $\boldsymbol{H}$. pertussis, Pr. vulgaris, Aerobacter aerogenes, Coryne. hoffmanni, Myco. phlei, Diplococcus pneumoniae, Esch. coli, B. subtilis, Past. boviseptica and an unidentified chromogenic coccus, were grown on the appro- 
priate medium and the cells removed and washed free from the medium by six successive washings with physiological saline of centrifuged deposits. The organisms were hydrolysed by refluxing for $40 \mathrm{hr}$. with $6 \mathrm{~N}-\mathrm{HCl}$. The hydrolysate was evaporated to dryness in vacuo and redissolved in a little distilled water. Single- and two-dimensional chromatograms showed the presence of the following amino-acids and groups of acids, which were present in all cases: cystine, aspartic acid, glutamic acid, serine, glycine, threonine, lysine, histidine, arginine, alanine, proline, the leucine group, the valine group, and tyrosine. No evidence of previously unknown amino-acids was obtained in any of the chromatograms. Although it is always possible that such acids may be present in bacteria, it is fairly clear that they could not be present in amounts greater than $2 \%$ of the total amino-acids. No gross differences between the amino-acid compositions of different species of bacteria were evident with this technique.

\section{DISCUSSION}

Partition paper chromatography makes possible a new approach to the study of the nitrogen metabolism of bacteria. This survey shows that it is possible to obtain a fairly complete picture of the amino-acid and polypeptide composition of bacterial filtrates, and to make comparative studies of large numbers of strains or species with great facility. Moreover, under reasonably standard conditions, the results are reproducible and many of the effects unequivocal.

It is, however, necessary to point out two limitations of our method which affect the interpretation of the results. First, in spite of the extreme sensitivity of the ninhydrin reaction, many of the changes which take place in bacterial cultures, such as the utilization of specific amino-acids as essential nutrients, occur at a level which is below the sensitivity of the method. Secondly, although the chromatogram records the presence or absence of ninhydrinpositive material it throws little direct light on the mechanism of the reactions which may be taking place.

There appears to be a general relationship between the nutrient requirements of an organism and the effect of growth on the amino-acid composition of the medium. Usually those organisms with simple nutrient requirements (capable of using ammonia) do not affect the chromatogram during the initial growth stage. This is illustrated in Pl. 1, fig. 2, with $B$. subtilis and Ps. aeruginosa.

The results with $P$ s. aeruginosa would suggest that in a mixture of ammonium salts and amino-acids organisms which can utilize the ammonium salts do so in preference to amino-acids. It is one of the advantages of the chromatographic method that the effects obtained in complex mixtures can be studied. It is also of interest to note that organisms with simple nutrient requirements often produce the most extensive changes in the chromatogram on prolonged incubation at alkaline or acid reaction.

With organisms having more complex nutrient requirements a change in the chromatogram always takes place during growth. The outstanding observation here is the universal utilization of serine. The preferential use of serine suggests that the amino-acid plays a prominent part in bacterial metabolism. The 
mechanism of the reaction and the reasons for the selection of this particular amino-acid will be studied further.

Besides serine, other amino-acids may be metabolized during and after the initial growth. Both species and group differences are apparent. Perhaps the most interesting is the difference between Gram-positive and Gram-negative organisms, illustrating once again the profound dissimilarity of these two groups of bacteria.

The synthesis of polypeptides during and after growth, the mechanism of their production and the function of the associated amino-acids, are of special interest. Polypeptide synthesis by bacteria has often been regarded as a rather unusual aspect of bacterial metabolism, little being recorded except the formation of some polypeptide antibiotics and the capsular substance of B. anthracis. It seems, however, that polypeptide synthesis may be a more general phenomenon in bacterial metabolism than hitherto recognized. Whether the ninhydrin-positive materials responsible for the unidentified spots are in all cases polypeptides remains to be seen. But it is clear that these ninhydrinpositive materials exhibit all grades of specificity, varying from the group specificity shown by the 'polypeptide' appearing in the aspartic acid position, which is synthesized by most Gram-negative bacteria and the two 'polypeptides' produced by all members of the genus Proteus, to the species-specific polypeptide of $\mathrm{Cl}$.tetani. Both in the removal of amino-acid and in the synthesis of 'polypeptide' group and species differences are apparent. These differences are likely to be of value in bacterial classification.

Using specific decarboxylase preparations Gale (1947) has shown that bacteria store a number of free amino-acids inside the cell. Preliminary observations with the chromatographic method confirm this, and show that other amino-acids are also stored. Taylor's (1947) observation that Grampositive, but not Gram-negative, organisms store glutamic acid is also confirmed (Pl. 2, fig. 3).

Contrary to Polson's (1948) observations, the amino-acid composition of hydrolysates of twelve species of bacteria examined by two-dimensional chromatography did not include any previously unknown amino-acids. 
Journal of General Microbiology, Vol. :3, No. 2

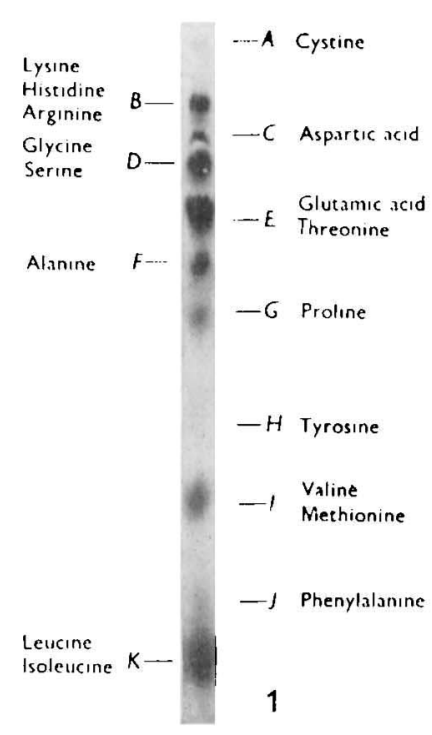

liij. 1

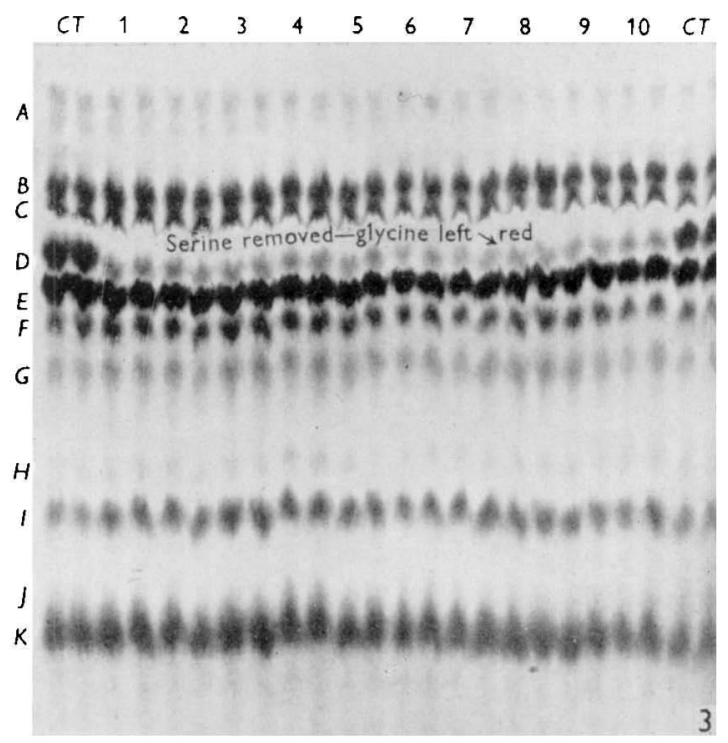

Fï. :3

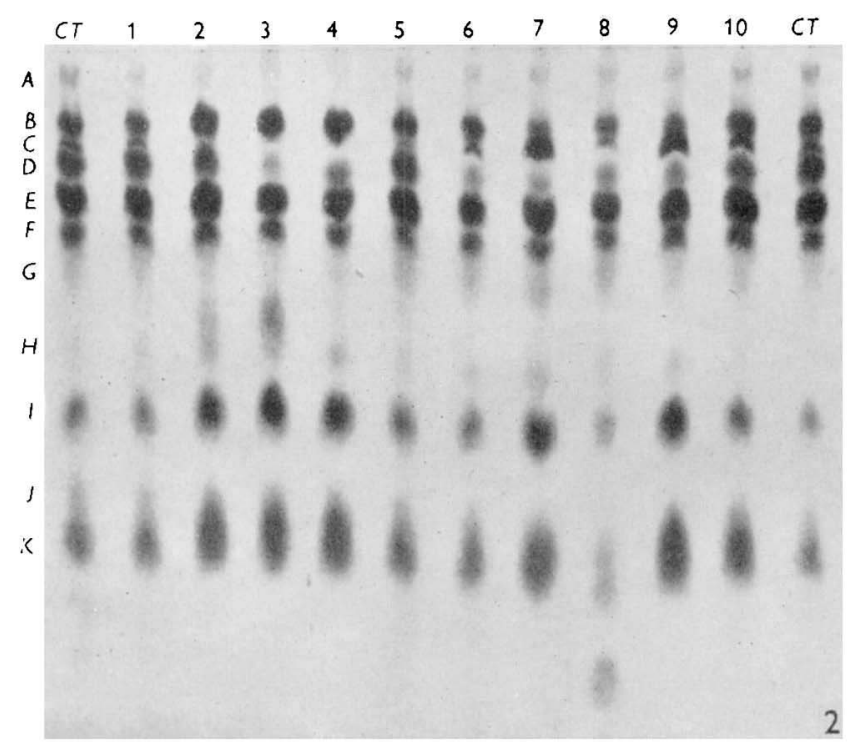

Fig. 2

H. Proom and A. J. Woiwod Nitrogen merabolism of Bacteria. Plate 1 
Journal of General Microbiology, Vol. 3, No. 2

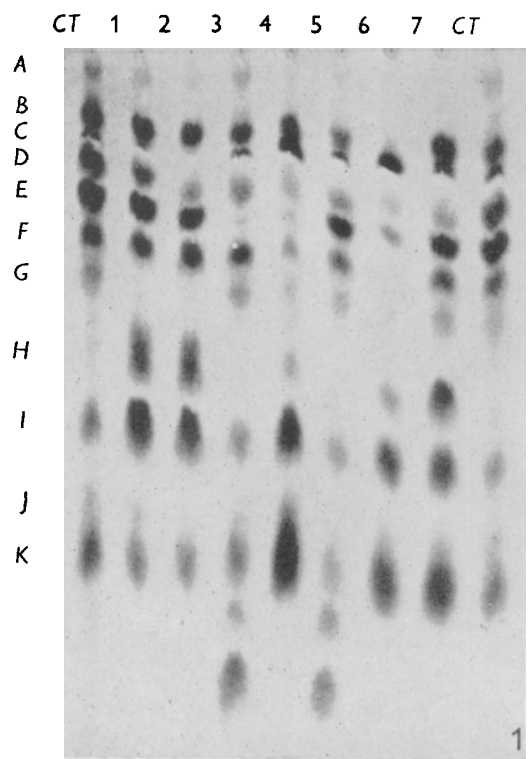

Fig. 1

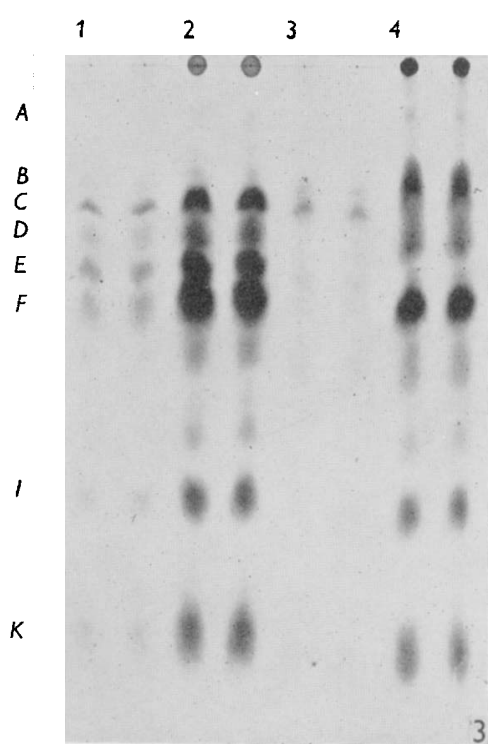

Fig. 3

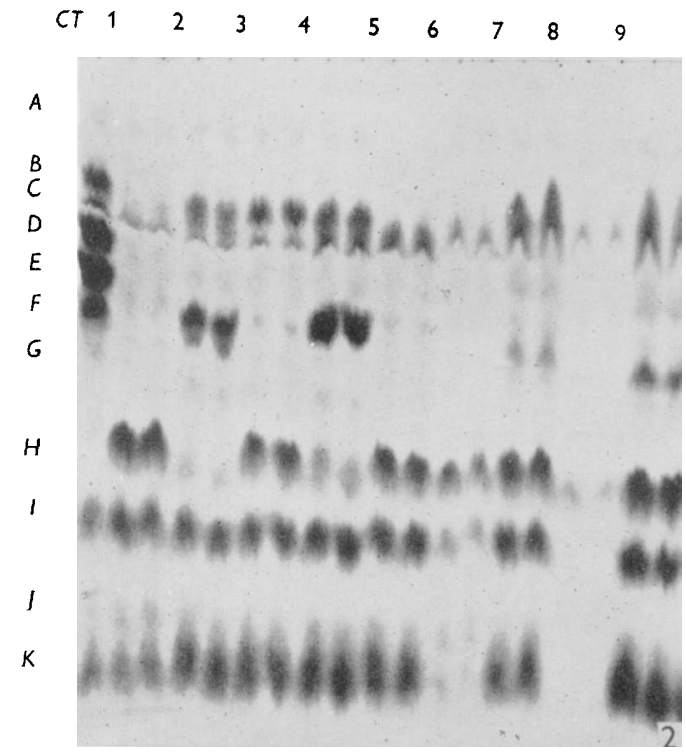

Fig. 2

H. Proom and A. J. Worwod-Nitrogen metabolism of bacteria. Plate 2 


\section{REFERENCES}

Consden, R., Gordon, A. H. \& Martin, A. J. P. (1944). Qualitative analysis of proteins: a partition chromatographic method using paper. Biochem. J. 41, 224.

Elliot, W. H. \& Gale, E. F. (1948). Glutamine-synthesizing system of Staphylococcus aureus: its inhibition by crystal violet and methionine-sulphoxide. Nature, Lond., 161, 129.

Gale, E. F. (1947). The assimilation of amino-acids by bacteria. 1. The passage of certain amino-acids across the cell wall and their concentration in the internal environment of Streptococcus faecalis. J. gen. Microbiol. $1,53$.

Polson, A. (1948). New amino-acids in bacterial hydrolysates. Nature, Lond., 161, 351.

TAYLOR, E. (1947). The assimilation of amino-acids by bacteria. 3. Concentration of free amino-acids in the internal environment of various bacteria and yeasts. J. gen. Microbiol. $1,86$.

Worwod, A. J. (1949). A technique for examining large numbers of bacterial culture filtrates by partition chromatography. J. gen. Microbiol. 3, 312.

\section{EXPLANATION OF PLATES}

\section{Plate 1}

Fig. 1. Key chromatogram of control medium showing the positions of the amino-acids on the chromatogram. The capital letters on the left of the chromatograms refer to this key.

Fig. 2. Chromatogram of 3-day filtrates from bacterial cultures: $C \boldsymbol{T}=$ Control medium (i.e. uninoculated). 1, B. subtilis (no change); 2, Str. pyogenes (decrease in aspartic acid and serine); 3, Staph. aureus (decrease in aspartic acid and serine and appearance of 'polypeptide'); 4, Coryne. diphtheriae (decrease in aspartic acid and serine); 5, Ps. aeruginosa (no change); 6, Sh. dysenteriae (decrease in serine); 7 , Ser. marcescens (decrease in serine, basic group, increase in aspartic acid); $8, \operatorname{Pr}$. vulgaris (decrease in serine, leucine and valine groups, appearance of 'polypeptide'); 9, Esch. coli (decrease in serine, increase in aspartic acid); 10, Past. boviseptica (decrease in serine).

Fig. 3. Chromatogram of 3-day filtrates from cultures of ten strains of Sh. paradysenteriae (Boyd types) showing decrease in serine and consistency of the effect. $C T=$ Control medium; $1-10=$ filtrates.

\section{Plate 2}

Fig. 1. Chromatogram of filtrates of cultures showing 'polypeptide' formation. $C T=$ control medium(i.e. uninoculated). 1, Cl.bifermentans ('polypeptide' above tyrosine and on valine group); 2, Cl. sordellii ('polypeptide' above tyrosine and on valine group); 3, Cl. tetani ('polypeptide 'below leucine group); 4, Ser. marcescens ("polypeptide' in aspartic acid position); 5, Pr. vulgaris ('polypeptide' below leucine group); 6, Esch. coli at alkaline reaction ('polypeptide' in aspartic acid position); 7, Sh. paradysenteriae ('polypeptide' just above tyrosine spot). $C T=$ control medium (i.e. uninoculated). Associated aminoacids : 1,2 , proline ; 3 , glutamic acid ; 4 , ? serine ; 5 , leucine and valine groups ; 6 , ? serine ; 7 , glutamic acid.

Fig. 2. Chromatogram of 10-day filtrates at acid reaction of nine strains of Esch. coli showing appearance of 'polypeptide' near tyrosine spot and the inverse relationship of the intensity of the glutamic acid and 'polypeptide' spots. Also the progressive removal of all ninhydrin-positive material from the chromatogram following prolonged incubation at acid reaction. $C T=$ control medium; $1-9$, filtrates.

Fig. 3. Chromatogram of the supernatants from washed, and also from washed and disintegrated cultures of Staph. aureus and Esch. coli showing the storage of amino-acids in the cell, the absence of polypeptides and the presence of glutamic acid in the disintegrate of Staph. aureus but not of Esch. coli. 1, washed Staph. aureus; 2, disintegrated Staph. aureus; 3, washed Esch. coli; 4, disintegrated Esch. coli. 
